nephron

Practice

\title{
UK Renal Registry 18th Annual Report: Chapter 12 Epidemiology of Reported Infections amongst Patients Receiving Dialysis for Established Renal Failure in England 2013 to 2014: a Joint Report from Public Health England and the UK Renal Registry
}

\author{
Rebecca Evans ${ }^{a}$, Fergus Caskey ${ }^{\mathrm{a}}$, Richard Fluck ${ }^{\mathrm{b}}$, Lisa Crowley ${ }^{c}$, John Davies $^{\mathrm{d}}$, \\ Olisaeloka Nsonwu ${ }^{d}$, Ken Farrington ${ }^{\mathrm{e}}$

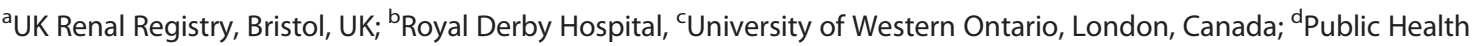 \\ England, London, UK; ' Lister Hospital, Stevenage, UK
}

\section{Key Words}

Clostridium Difficile · Dialysis · Epidemiology · Escherichia Coli · Established renal failure - Infection · MRSA · MSSA - Staphylococcus

\section{Summary}

- From 1st May 2013 to 30th April 2014 there were 35 episodes of Methicillin resistant Staphylococcus aureus (MRSA) bacteraemia in established renal failure patients on dialysis.

- This is now fairly stable year-on-year equating to a rate of 0.15 episodes per 100 dialysis patient years, following an initial decline in rates from 4.0 episodes per 100 dialysis patient years in 2005 when reporting began.

- Methicillin sensitive Staphylococcus aureus (MSSA) bacteraemia rates were slightly higher this year at 2.23 per 100 dialysis patient years (compared with 1.59 episodes per 100 dialysis patient years last year) with 526 episodes of blood stream infection reported. In 2005, the first year this was reported, there were 1,114 MSSA bacteraemias in 54 centres.

- There were 247 Clostridium difficile infection episodes with a rate of 1.05 per 100 dialysis patient years, slightly higher than last year at 0.55 episodes per 100 dialysis patient years.

- Escherichia coli infections occurred at a rate of 1.49 per 100 dialysis patient years, very similar to the rate reported last year (1.32 episodes per 100 dialysis patient years).

- This report has utilised a new methodology to identify cases, linking all established renal failure cases known to the UK Renal Registry (UKRR) with all infections reported to Public Health England and avoids the need for the local microbiology team to flag the patient as a renal patient. This may have increased the reliability of diagnosis at the UKRR level.

- In each infection for which access data were collected, the presence of a central venous catheter appeared to correlate with increased risk.

\section{KARGER}

Fax +4161306 1234 E-Mail karger@karger.com www.karger.com/nef
(C) 2016 The UK Renal Registry Published by S. Karger AG, Basel $1660-8151 / 16 / 1325-0279 \$ 39.50 / 0$

Karger Open access

This article is licensed under the Creative Commons AttributionNonCommercial-NoDerivatives 4.0 International License (CC BYNC-ND) (http://www.karger.com/Services/OpenAccessLicense). Usage and distribution for commercial purposes as well as any distribution of modified material requires written permission. 


\section{Introduction}

Infection remains the second leading cause of death in patients with established renal failure (ERF) who received renal replacement therapy (RRT). The high rates of systemic infection reported in haemodialysis (HD) patients are related to their impaired immune system, the high number of invasive procedures they are exposed to and the type of vascular access used [1]. This report covers one year of reporting for Methicillin resistant Staphylococcus aureus (MRSA), Methicillin sensitive Staphylococcus aureus (MSSA), Escherichia coli (E. coli) bloodstream infections (BSI) and Clostridium difficile infections (CDI) in adult patients with ERF who were receiving dialysis in England.

Previous UK Renal Registry (UKRR) reports have detailed the epidemiology of Staphylococcus aureus bacteraemias, E. coli BSIs and CDIs in patients with ERF receiving dialysis [2]. As well as the mandatory reporting of MRSA BSIs, reporting of MSSA has been mandated since January 2011 and E. coli BSIs since June 2011; CDI reporting has been mandatory for all patients aged two and above since 2007. CDIs are reported according to a national testing protocol although during the timeframe of this report there may have been some inter-hospital variation in testing approaches [3].

The data were supplied by clinical staff and captured using a secure web-based system, the Healthcare Associated Infection Data Capture System (HCAI-DCS). Previous reports have confirmed that whilst dialysis patients remained at increased risk from MRSA there has been a continued year on year decline in the number of reported episodes of bacteraemia [2].

\section{Methods}

This report covers the period of 1st May 2013 to 30th April 2014. It should be noted that although reporting is mandatory for these data collections (MRSA, MSSA and E. coli BSI and $\mathrm{CDI}$ ), completion of documentation of information relating to renal failure is currently conducted on a voluntary basis depending on the data entry policy within the reporting NHS acute Trust. The methods used for the reporting of infections to Public Health England (PHE) have been described in previous UKRR reports (see appendix 1) [4,5].

In last year's report the number of alterations made by renal centres varied considerably and the extent to which this reflected differences in the accuracy of PHE data for their renal centre was not known [2]. This year to standardise the case identification process and minimise the number of alterations made by centres, for the first time UKRR data were used to identify adult patients with ERF who were receiving dialysis. This meant that identification was not dependent on the reporting of dialysis status by individual NHS acute Trusts via PHE's HCAI-DCS. A list of all adult patients identified in the UKRR database as receiving dialysis between 1st May 2013 and 30th April 2014 was sent to PHE for identification of bacteraemias and CDI associated with these patients. Records of positive blood cultures of the identified patients were then passed back to the UKRR. As this was the first year that the UKRR data was linked to PHE data for identification of infectious episodes in patients receiving dialysis the additional validation and data capture step was again implemented to ensure all records were accurately captured and completed. This additional validation step involved emailing clinical or infection control leads in the renal centre with the records reported to $\mathrm{PHE}$ and requesting they complete the following actions:

1 Confirm that each of the cases in the PHE file was correct, i.e. that it related to a dialysis patient receiving treatment at their centre at the time of the infection and

a Remove any cases that occurred in patients not on dialysis and receiving treatment at their centre at the time of the infection

b Add any cases that were not known to PHE but occurred in patients on dialysis and receiving treatment at their centre at the time of the infection

2 For all MRSA and MSSA cases, to confirm details on the dialysis modality and provide details on access in use at the time of the infection.

PHE report positive blood cultures as opposed to infectious episodes. For this report repeatedly positive blood cultures in the same individual within four weeks were treated as the same episode, beyond four weeks they were treated as new or re-infection. This additional step was implemented by the UKRR after the centre validation process. This is slightly more conservative than the approach taken for the Renal Indicator Dashboard, which defines separate infections as being positive cultures more than two weeks apart.

Centre-specific rates for each infection are presented per 100 dialysis patient years. The denominator for this rate was calculated at each centre by summing the number of days that every adult dialysis patient contributed between the 1st May 2013 and 30th April 2014. For example, a patient who started dialysis on the 1st April 2014 and remained on dialysis until at least the 30th April 2014 would contribute 30 days to the total. Similarly, when calculating the modality specific rates, the number of days that every dialysis patient spent on each modality during the collection period was summed. Number of patient years at risk by access type was estimated using data from the 2013 dialysis access audit. The percentage of prevalent patients on each form of vascular access on 31st December 2013 was multiplied by the total number of patients on HD on 31st December 2013 to give an estimate of the overall number of patient years at risk.

In order to adjust for variation in precision of estimated rate, the rate of bacteraemia/CDI per 100 dialysis patient years has been plotted against the centre size in a funnel plot. This process has been repeated for each infection. In the case of MRSA, a comparative box plot to demonstrate the overall trend is also shown. Table 12.1 lists the summary of audit measures stated in the Renal Association clinical practice guidelines. 
Table 12.1. Summary of all audit measures stated in Renal Association (RA) clinical practice guidelines relating to infection

\begin{tabular}{llc}
\hline RA audit measure & Reported & Reason for non-inclusion \\
\hline $1 \quad \begin{array}{l}\text { Centres should audit all Staphylococcus aureus bacteraemia (MRSA and MSSA) } \\
\text { episodes recorded as episodes per } 100 \text { patient years or episodes per 100 catheter } \\
\text { days or episodes per 100 AVF years }\end{array}$ & Yes \\
$2 \quad \begin{array}{l}\text { The annual Staphylococcus aureus bacteraemia rate should be less than } 2.5 \\
\text { episodes per 100 HD patients and less than 1.0 for MRSA over two years }\end{array}$ & Yes \\
$\begin{array}{l}\text { Centres should audit all episodes of Clostridium Difficile toxin (CDT) and express } \\
\text { rates as per } 100 \text { patient years }\end{array}$ & Yes \\
$\begin{array}{l}\text { Data should be collected on all episodes of VRE and ESBL bacteraemia episodes } \\
\text { per } 100 \text { patient years }\end{array}$ & Partly & $\begin{array}{c}\text { Only data on E. coli received } \\
\text { from PHE }\end{array}$ \\
\hline
\end{tabular}

$\mathrm{ESBL}=$ Extended-Spectrum betaLactamase; VRE = vancomycin-resistant enterococci

\section{Results}

\section{Validation}

This was the first year that UKRR data were used to identify patients with ERF who were receiving dialysis to link with PHE data and the second year that the UKRR performed the additional validation and data capture step in which centres were requested to add any additional episodes which were not captured by PHE. Table 12.2 displays the number of positive blood cultures reported to $\mathrm{PHE}$ and the changes to the data that occurred during the validation process. The majority of episodes were rejected because the patient was not receiving dialysis for established renal failure at the time of the infection e.g. they were an acute dialysis patient or a transplant patient at the time of infection. (Acute dialysis patients will be included from January 2016.) The majority of additions were cases which were not known to PHE. There were a number of positive

Table 12.2. Number of infectious episodes reported to Public Health England (PHE) and validated by renal centres

\begin{tabular}{lcccc}
\hline & MRSA & MSSA & CDI & E. coli \\
\hline $\begin{array}{l}\text { Number of positive blood } \\
\text { cultures reported to PHE }\end{array}$ & 37 & 565 & 242 & 381 \\
$\begin{array}{l}\text { Number of episodes rejected } \\
\text { by centres during validation }\end{array}$ & 0 & 3 & 2 & 5 \\
$\begin{array}{l}\text { Number of episodes added by } \\
\text { centres during validation }\end{array}$ & 2 & 3 & 11 & 1 \\
$\begin{array}{l}\text { Number of duplicate episodes } \\
\text { removed }\end{array}$ & 4 & 39 & 4 & 25 \\
$\begin{array}{l}\text { Total number of episodes } \\
\text { after validation process }\end{array}$ & 35 & 526 & 247 & 352 \\
\hline
\end{tabular}

Epidemiology of infection in dialysis patients blood cultures reported to PHE which related to one infectious episode, these were removed during the data validation step.

There was some variation in the response from centres to the validation process with some centres adding additional episodes, and other centres not adding any. However the number of alterations made by renal centres was considerably lower than in the previous year's report, with 147 episodes added by centres during validation last year compared with 17 episodes added by centres this year [2].

\section{Methicillin resistant Staphylococcus aureus}

Thirty-five MRSA bacteraemias were recorded as being associated with dialysis patients during the time frame of this report, at a rate of 0.15 (95\% CI 0.10 0.21 ) per 100 dialysis patient years (table 12.3). This rate was similar to the rate of 0.13 per 100 patients reported last year. In previous years there has been a steady reduction in the MRSA rates which this year appears to have plateaued (figure 12.1). However, this year for the first time the identification of cases did not rely on local flagging, so an actual continued reduction in MRSA cannot be ruled out. The modality in use at the time of infection was completed for all episodes but statistically valid comparisons between the modalities are difficult due to small numbers.

Centre level data can be seen in table 12.4 and includes the absolute number of episodes and rates per 100 dialysis patient years. The majority of centres did not report any MRSA bacteraemia episodes and only one centre had an infection rate in excess of 1 per 100 dialysis patient years. Figure 12.2 plots each centre's estimated rate against the number of patient years to take into account the greater variation expected as centre size decreases. The extremely

Nephron 2016;132(suppl1):279-288 
Table 12.3. Number and rate of infectious episodes in patients with established renal failure between $1 / 05 / 2013$ and 30/04/2014, by modality

\begin{tabular}{|c|c|c|c|c|}
\hline & \multicolumn{4}{|c|}{ Infection } \\
\hline & MRSA & MSSA & CDI & E. coli \\
\hline \multicolumn{5}{|c|}{ Number of episodes } \\
\hline Total & 35 & 526 & 247 & 352 \\
\hline HD & 32 & 514 & 222 & 333 \\
\hline PD & 3 & 12 & 25 & 19 \\
\hline \multicolumn{5}{|c|}{ Rate $(95 \%$ CI) per 100 patient years } \\
\hline Total & $0.15(0.10-0.21)$ & $2.23(2.05-2.43)$ & $1.05(0.92-1.19)$ & $1.49(1.34-1.66)$ \\
\hline HD & $0.16(0.11-0.22)$ & $2.53(2.32-2.76)$ & $1.09(0.96-1.25)$ & $1.64(1.47-1.83)$ \\
\hline $\mathrm{PD}$ & $0.09(0.02-0.27)$ & $0.37(0.19-0.64)$ & $0.77(0.50-1.13)$ & $0.58(0.35-0.91)$ \\
\hline
\end{tabular}

$\mathrm{HD}=$ haemodialyis; $\mathrm{PD}=$ peritoneal dialysis

low numbers of episodes at each centre makes the comparison of rates uncertain.

The Renal Association (RA) audit standard states that the annual MRSA rate should be less than 1.0 per $100 \mathrm{HD}$ patients averaged over two years. Figure 12.3 displays a funnel plot of MRSA rate per 100 prevalent $\mathrm{HD}$ patients across the two year period from 1st May 2012 to 30th April 2014. Only one centre had a rate higher than this standard.

\section{Methicillin sensitive Staphylococcus aureus}

In total, 526 episodes of MSSA bacteraemia were recorded in the period covered by this report, at a rate of 2.23 per 100 dialysis patient years (95\% CI 2.052.43). This was higher than last year's rate of 1.59 per 100 dialysis patient years. One centre did not report any MSSA episodes and the highest reported rate was 5.63 per 100 dialysis patient years (table 12.4). Based on

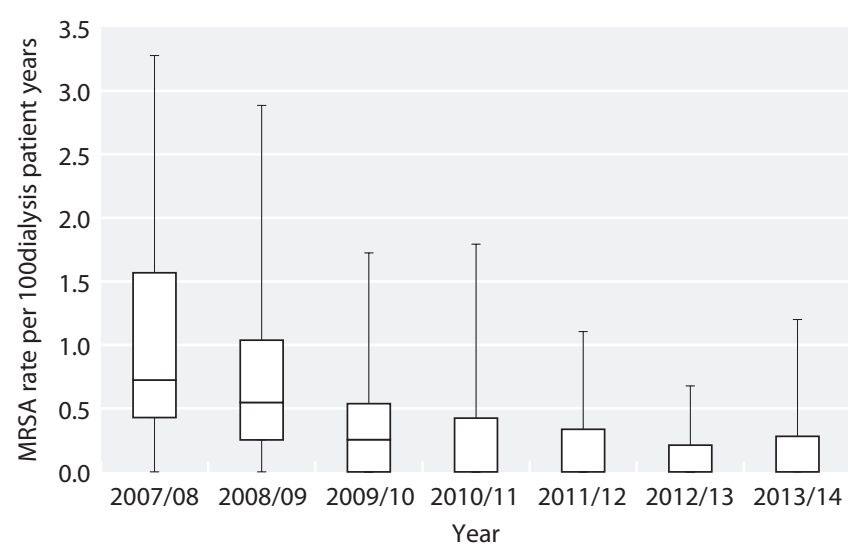

Fig. 12.1. Box and whisker plot of renal centres' MRSA rates per 100 dialysis patient years by reporting year

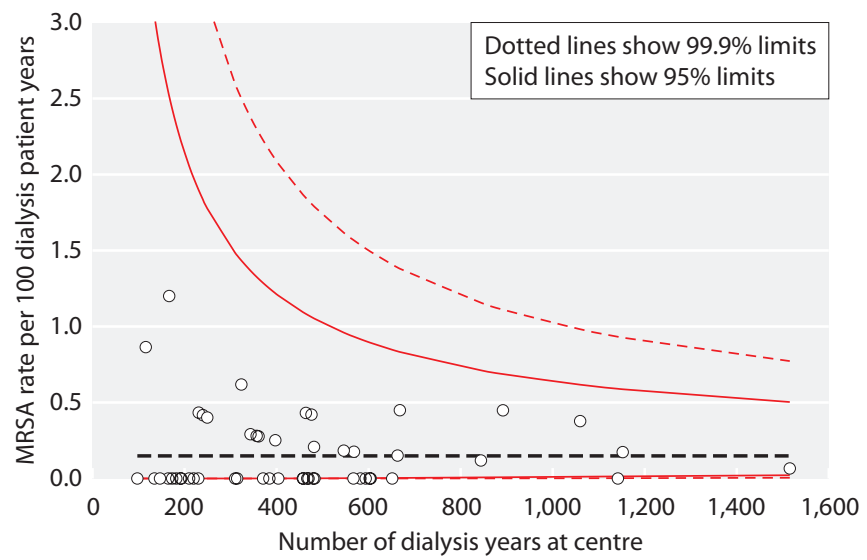

Fig. 12.2. Funnel plot of the MRSA bacteraemia rate per 100 dialysis patient years by renal centre, 1st May 2013 to 30th April 2014

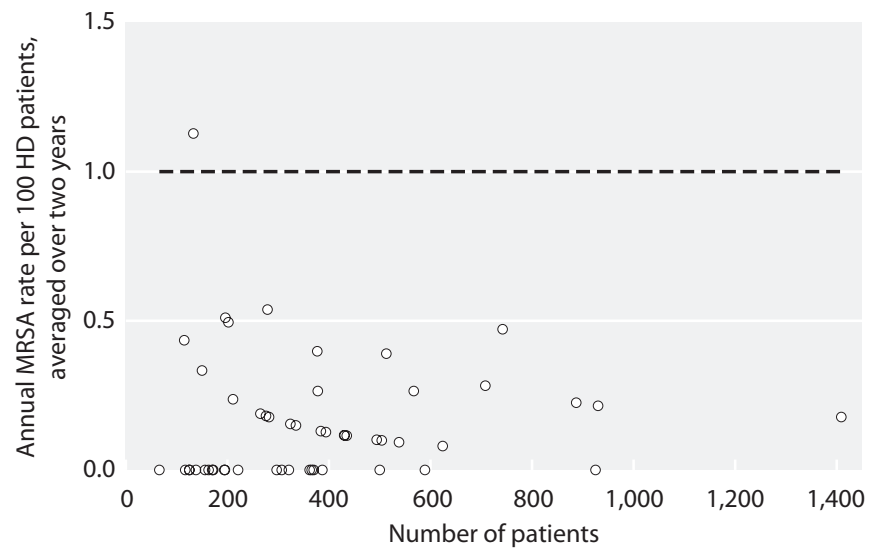

Fig. 12.3. Funnel plot of the MRSA bacteraemia two-year rate per 100 prevalent HD patients, 1st May 2012 to 30th April 2014 Dotted line depicts Renal Association standard 
Table 12.4. Number and rate of infectious episodes in patients with established renal failure by renal centre

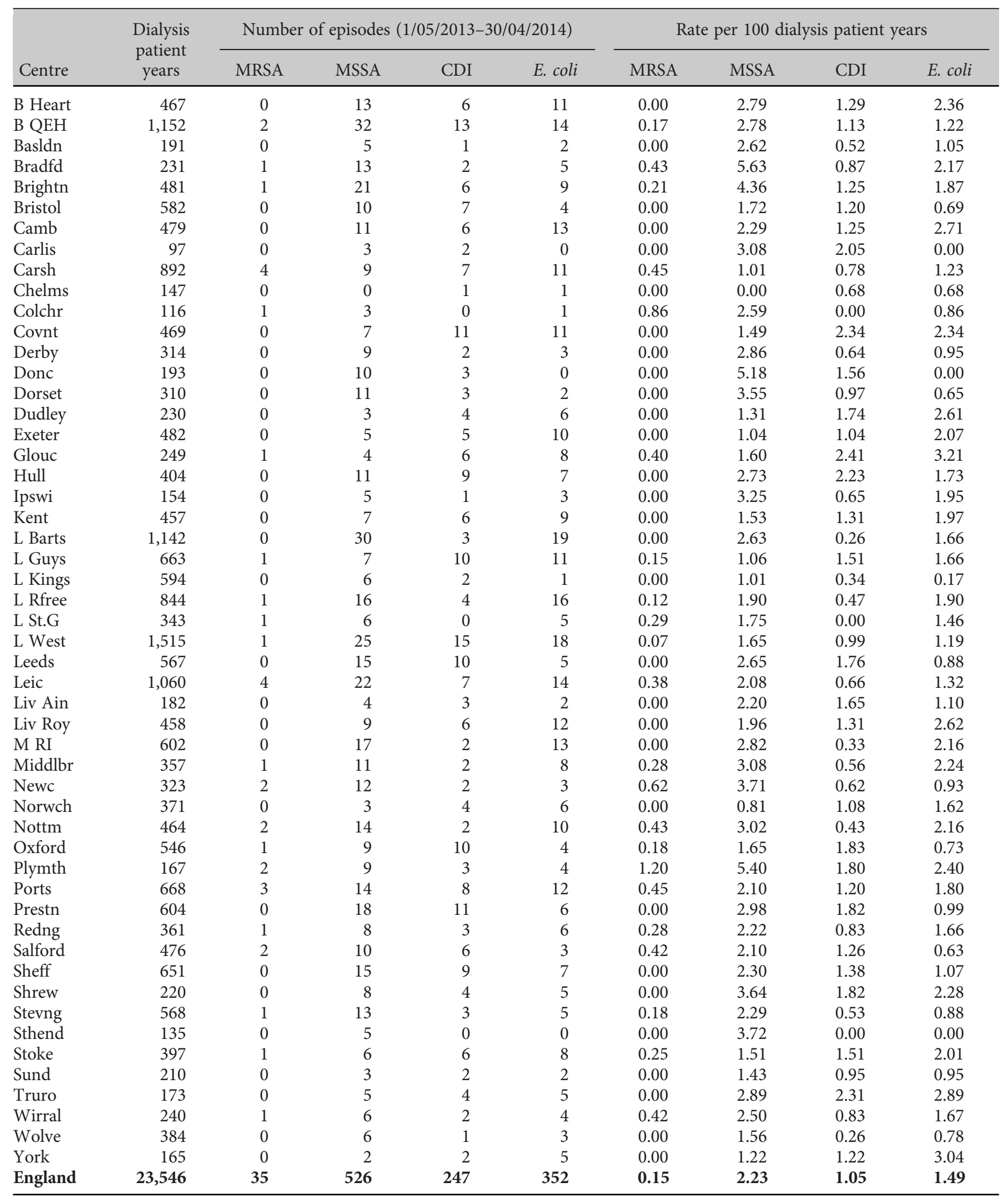




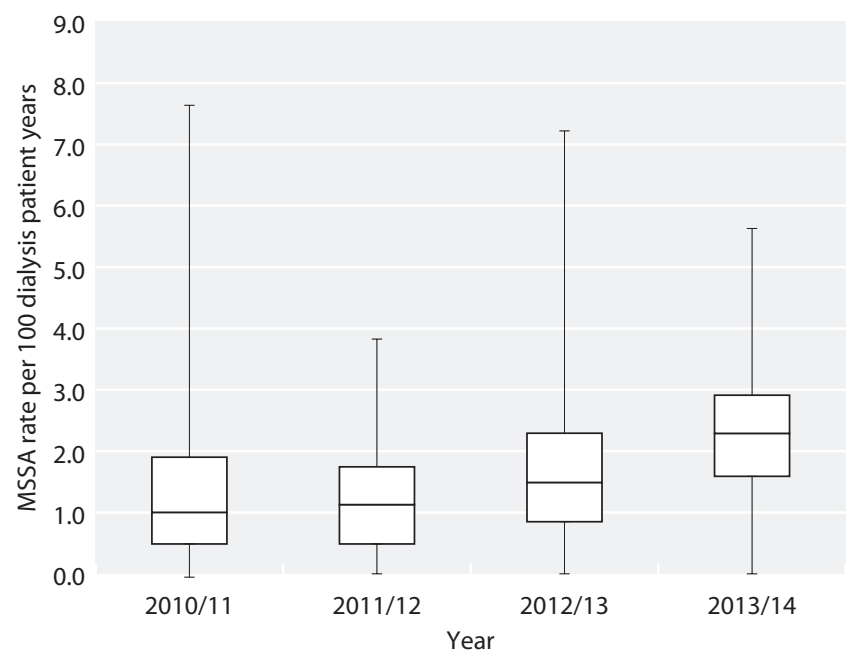

Fig. 12.4. Box and whisker plot of renal centres' MSSA rates per 100 dialysis patient years by reporting year

the reported data, $46 \%$ of centres in England reported rates of MSSA higher than the Renal Association standard. The rates have remained fairly steady over the past three years although with a slight increasing trend (figure 12.4). Again, caution must be exercised when making year on year comparisons as the apparent variation in rates may be a reflection on the differences in the way the data has been collected and validated over the years, with the data validation step by centres adopted in the past two years and UKRR data linked with PHE data for the first time this year. Figure 12.5 plots each centre's estimated rate against the number of patient years to take into account the greater variation expected as centre size decreases.

The peritoneal dialysis (PD) cohort had a lower rate of MSSA bacteraemia per 100 patient years than the HD cohort $(0.37,95 \%$ CI $0.19-0.64$ compared with 2.53, 95\% CI 2.32-2.76) (table 12.3).

\section{Type of dialysis access and infection}

There were major variations in the number of episodes of both MRSA and MSSA bacteraemia according to access type. Patients dialysing through a central venous catheter (CVC) at the time of the infection were subject to more episodes of bacteraemia than those with other types of access (table 12.5). Absolute rates cannot be calculated because vascular access has until now only been captured at one point every 12 months, so the time at risk on each form of access was not available. The estimated number of patient years at risk is provided only as an estimate of the time at risk and rates derived from this should be treated with caution. It is based on

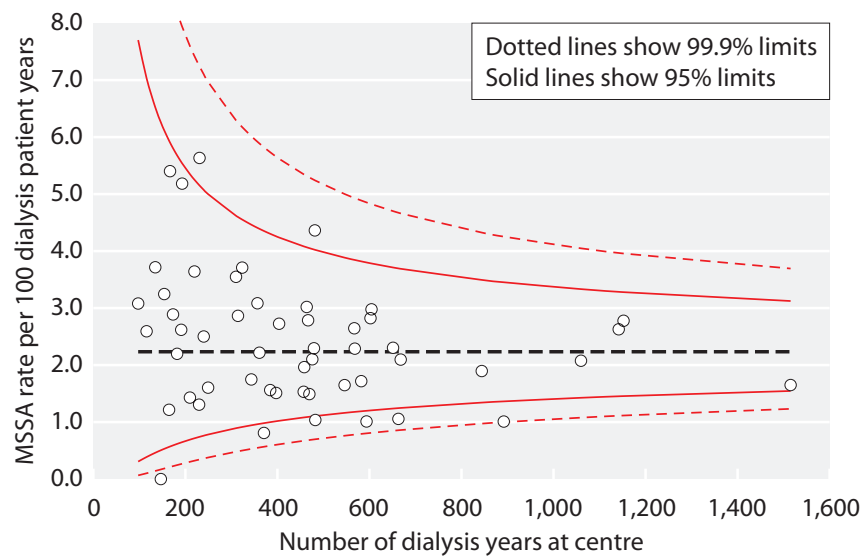

Fig. 12.5. Funnel plot of the MSSA bacteraemia rate per 100 dialysis patient years by renal centre, 1st May 2013 to 30th April 2014

the distribution of access types using data on the 33 centres in England who provided prevalent access data in the 2013 dialysis access audit return. This distribution was then applied to the total number of patients on HD in England on 31st December 2013 to give an overall estimate for England.

\section{Clostridium difficile}

In total, 247 episodes of CDI were recorded in the period covered by this report, at a rate of 1.05 (95\% CI $0.92-1.19)$ per 100 dialysis patient years. Based on the reported data, this was higher than last year's rate of 0.55 per 100 dialysis patient years, however this may be a reflection on the change in the way the data has been collected and validated this year. Three centres did not report any CDI episodes and the highest reported rate was 2.41 per 100 dialysis patient years (table 12.4). Figure 12.6 plots each centre's estimated rate against

Table 12.5. Type of dialysis access in use at the time of infection for HD patients

\begin{tabular}{lrrrrr}
\hline & \multicolumn{5}{c}{$\begin{array}{c}\text { Number of episodes } \\
(1 / 05 / 2013-30 / 04 / 2014)\end{array}$} \\
\cline { 2 - 6 } & AVF & AVG & CVC & PD & No data \\
\hline $\begin{array}{l}\text { Estimated number of } \\
\text { patient years at risk }\end{array}$ & 14,492 & 850 & 4,754 & 3,176 & \\
MRSA & 6 & 4 & 21 & 3 & 1 \\
MSSA & 183 & 36 & 250 & 10 & 47 \\
\hline
\end{tabular}

$\mathrm{AVF}=$ arteriovenous fistula; $\mathrm{AVG}=$ arteriovenous graft; $\mathrm{CVC}=$ central venous catheter; $\mathrm{PD}=$ peritoneal dialysis 


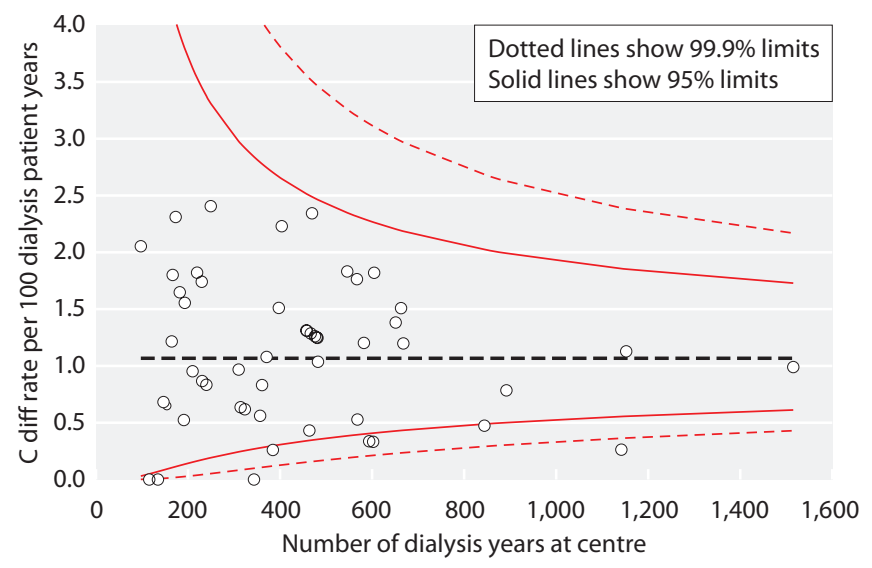

Fig. 12.6. Funnel plot of the CDI rate per 100 dialysis patient years by renal centre, 1st May 2013 to 30th April 2014

the number of patient years to take into account the greater variation expected as centre size decreases. Rates were higher in the HD than the PD cohort $(1.09,95 \%$ CI 0.96-1.25 compared with 0.77, 95\% CI 0.50-1.13, respectively) (table 12.3).

\section{Escherichia coli}

A total of 352 episodes of E. coli bacteraemia were recorded in the period covered by this report, at a rate of 1.49 per 100 dialysis patient years (95\% CI $1.34-$ 1.66). This was slightly higher than last year's rate of 1.32 per 100 dialysis patient years, however this may be a reflection on the change in the way the data has been collected and validated this year.

Centre level data are displayed in table 12.4 with considerable between-centre variation in $E$. coli bacteraemia

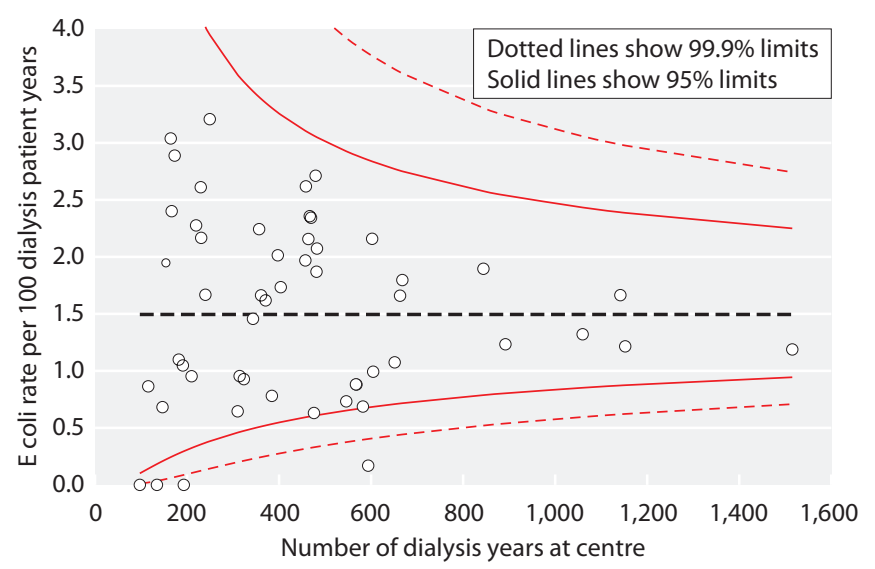

Fig. 12.7. Funnel plot of the Escherichia coli bacteraemia rate per 100 dialysis patient years by renal centre, 1st May 2013 to 30th April 2014 rates. Three centres did not report any episodes and the highest reported rate was 3.21 per 100 dialysis patient years. Figure 12.7 plots each centre's estimated rate against the number of patient years to take into account the greater variation expected as centre size decreases.

Here too, PD was associated with a lower rate of infection per 100 patient years than HD $(0.58,95 \% \mathrm{CI}$ 0.35-0.91 compared with $1.64,95 \%$ CI 1.47-1.83, respectively) (table 12.3).

\section{Conclusions}

This report has presented data from one year of infections in adult ERF patients receiving dialysis and extends the work done in previous reports from Public Health England and the UK Renal Registry [2]. In previous reports the numbers and rates of MRSA BSIs in dialysis patients had fallen. However this year the rate has remained similar to that of last year. This change has mirrored the general improvement in MRSA rates seen across England over the same time period. General measures have included increased training, awareness and screening. In addition, there are dialysis specific factors that have led to improvement. These include enhanced screening programmes and increased attention to care of access. Despite the change in the reporting mechanism this sustained improvement is welcome.

This report also presents the third full year of reporting of MSSA bacteraemia episodes although MSSA was reported in the 2005 vascular access report. The rate of MSSA bacteraemia remained significantly higher than for MRSA with a 15 fold increased reporting rate. When Staphylococcal aureus infections were first reported in the 2004 cohort about $1 / 3$ were due to MRSA. This change in pattern of resistance requires further study.

The presence of a central venous catheter remained a significant risk factor for MSSA bacteraemia when compared to an arteriovenous fistula. However, there were a significant number of MSSA infections in people using an AVF. This study is limited in determining whether an infection was a direct consequence of the access and there are no data on outcomes. The discrepancy between the rates of MRSA and MSSA is notable and suggests that MSSA continues to be a significant issue in the dialysis population. A recent meta-analysis suggested that the use of mupirocin is associated with a reduced risk of 
bacteraemia in a screened population but practice within the UK may vary considerably [6]. For example, a single centre UK study suggested that eradication can be effective in just $36 \%$ of individuals but is associated with a reduced risk of MSSA bacteraemia in those who do respond [7]. Screening programmes, eradication therapy and access care policies for both CVC and AVF may vary between centres. Patients remained vulnerable to MSSA and a study of practice patterns may yield useful insights to improve care.

Data availability on CDI and E. Coli are relatively new. The survey this year did not ask for data on access for these episodes but may be of indirect relevance. The report again demonstrates centre variation. The reasons for this are not immediately clear. CDI risk may be associated with antibiotic exposure and data on centre antibiotic usage may be useful. E. coli bacteraemia is also relatively frequent. Further, there is nearly a three fold increased risk of $E$. Coli bacteraemia in HD compared to PD patients and while this could reflect haemodynamic stress and gut translocation in HD patients [8], it could also simply reflect the fact that HD patients tend

\section{References}

1 Bray BD, Boyd J, Daly C, Donaldson K, Doyle A, Fox JG, et al. Vascular access type and risk of mortality in a national prospective cohort of haemodialysis patients. QJM - an International Journal of Medicine. 2012;105(11):1097-103

2 https://www.gov.uk/government/uploads/system/uploads/attachment_ data/file/215135/dh_133016.pdf

3 Pitcher D, Rao A, Caskey F, Davies J, Crowley L, Fluck R, Farrington K. UK Renal Registry 17th Annual Report: Chapter 12 Epidemiology of Reported Infections amongst Patients Receiving Dialysis for Established Renal Failure in England in 2012 to 2013: a Joint Report from Public Health England and the UK Renal Registry. Nephron 2015;129 (suppl 1):257-265

4 Fluck R, Wilson J, Tomson CRV. UK Renal Registry 12th Annual Report (December 2009): Chapter 12 Epidemiology of Methicillin Resistant Staphylococcus Aureus Bacteraemia Amongst Patients Receiving Dialysis for Established Renal Failure in England in 2008: a joint report from the UK Renal Registry and the Health Protection Agency. Nephron Clinical Practice. 2010;115:C261-C70 to be frailer and that PD is contraindicated when there is significant bowel disease.

The introduction of the data linkage between PHE and UKRR this year has contributed to improved data accuracy and completeness of the data. It has minimised the data collection burden on centres by minimising the number of alterations required by centres during the data validation step. Consistency of data collection, validation and reporting in future years will enable trends to be more clearly identified. However, there is a need to interpret variation between centres by exploring practice patterns and thereby improve care.

Conflicts of interest: the authors declare no conflicts of interest

\section{Acknowledgements}

The authors wish to acknowledge the help of our colleagues at renal centres across the country for their assistance in compiling this report.
5 Fluck R, Wilson J, Davies J, Blackburn R, O’Donoghue D, Tomson C. UK Renal Registry 11th Annual Report: Chapter 12 Epidemiology of Methicillin Resistant Staphylococcus aureus bacteraemia amongst patients receiving Renal Replacement Therapy in England in 2007. Nephron Clinical Practice. 2009;C247-C56

6 Grothe C1, Taminato M, Belasco A, Sesso R, Barbosa D. Screening and treatment for Staphylococcus aureus in patients undergoing hemodialysis: a systematic review and meta-analysis. BMC Nephrol. 2014;15:202. doi: 10.1186/1471-2369-15-202

7 Price A, Sarween N, Gupta I, Baharani J. Meticillin-resistant Staphylococcus aureus and meticillin-susceptible Staphylococcus aureus screening in a cohort of haemodialysis patients: carriage, demographics and outcomes. J Hosp Infect. 2015;90(1):22-7. doi: 10.1016/j.jhin.2015.01.001

8 McIntyre CW1, Harrison LE, Eldehni MT, Jefferies HJ, Szeto CC, John SG, Sigrist MK, Burton JO, Hothi D, Korsheed S, Owen PJ, Lai KB, Li PK. Circulating endotoxemia: a novel factor in systemic inflammation and cardiovascular disease in chronic kidney disease. Clin J Am Soc Nephrol. 2011;6(1):133-41. doi: 10.2215/CJN.04610510 


\section{Appendix 1}

Processes for reporting of infections to Public Health England

All cases of Methicillin Resistant Staphylococcus aureus (MRSA), Methicillin Susceptible Staphylococcus aureus (MSSA), Escherichia coli and Clostridium difficile which satisfy the criteria below are reported via the Healthcare Associated Infection Data Capture System (HCAI-DCS) which is a real-time, secure web enabled system. Criteria for what constitutes an infection are as follows:

1 MRSA bacteraemia: The following MRSA positive blood cultures must be reported to PHE:

All cases of MRSA bacteraemia caused by $S$. aureus resistant to methicillin, oxacillin, cefoxitin or flucloxacillin. Further details on surveillance of MRSA bacteraemia in patients with renal disease are available online [1].

All reported MRSA bacteraemia are subject to a post infection review [2]. The included renal data includes all cases regardless of whether they were assigned to a Trust, CCG or Third party via the PIR process.

2 MSSA bacteraemia: The following MSSA positive blood cultures must be reported to PHE:
All cases of MSSA bacteraemia caused by $S$. aureus which are not resistant to methicillin, oxacillin, cefoxitin, or flucloxacillin i.e. not subject to MRSA reporting.

3 E. coli bacteraemia: The following E. coli positive blood cultures must be reported to PHE:

All laboratory confirmed cases of $E$. coli bacteraemia

4 C. difficile Infection: Any of the following defines a C. difficile infection case in patients aged 2 years and above and must be reported to PHE [3]:

a Diarrhoeal stools (Bristol Stool types 5-7) where the specimen is $C$. difficile toxin positive.

b Toxic megacolon or ileostomy where the specimen is $C$. difficile toxin positive.

c Pseudomembranous colitis revealed by lower gastro-intestinal endoscopy or Computed Tomography.

d Colonic histopathology characteristic of $C$. difficile infection (with or without diarrhoea or toxin detection) on a specimen obtained during endoscopy or colectomy.

e Faecal specimens collected post-mortem where the specimen is $C$. difficile toxin positive or tissue specimens collected post-mortem where pseudomembranous colitis is revealed or colonic histopathology is characteristic of $C$. difficile infection.

\section{References}

1 http://webarchive.nationalarchives.gov.uk/20140714084352/http://www. hpa.org.uk/webc/HPAwebFile/HPAweb_C/1194947399620

2 http://www.england.nhs.uk/wp-content/uploads/2014/04/mrsa-pir-guid-

3 https://www.gov.uk/government/uploads/system/uploads/attachment_ data/file/215135/dh_133016.pdf april14.pdf 
